\title{
Demonstration of Phase-conjugated Subcarrier Coding for Fiber Nonlinearity Compensation in CO-OFDM Transmission
}

\author{
Son Thai Le, Mary E. McCarthy, Naoise Mac Suibhne, Mohammad A. Z. Al-Khateeb, \\ Elias Giacoumidis, Nick Doran, Andrew D. Ellis and Sergei K. Turitsyn
}

\begin{abstract}
In this paper, we demonstrate through computer simulation and experiment a novel subcarrier coding scheme combined with pre-electrical dispersion compensation (pre-EDC) for fiber nonlinearity mitigation in coherent optical orthogonal frequency division multiplexing (CO-OFDM) systems. As the frequency spacing in CO-OFDM systems is usually small (tens of MHz), neighbouring subcarriers tend to experience correlated nonlinear distortions after propagation over a fiber link. As a consequence, nonlinearity mitigation can be achieved by encoding and processing neighbouring OFDM subcarriers simultaneously. Herein, we propose to adopt the concept of dual phase conjugated twin wave (dual PCTW) for CO-OFDM transmission. Simulation and experimental results show that this simple technique combined with $50 \%$ pre-EDC can effectively offer up to $1.5 \mathrm{~dB}$ and $0.8 \mathrm{~dB}$ performance gains in CO-OFDM systems with BPSK and QPSK modulation formats, respectively.
\end{abstract}

Index Terms -Nonlinearity mitigation, coherent detection, coherent optical transmission, orthogonal frequency division multiplexing.

\section{INTRODUCTION}

$\mathrm{Th}$ he nonlinear impairment due to Kerr effect limits the maximum signal power that could be launched into an optical fiber, without degrading the effective signal-to-noise (SNR) ratio or the system performance [1-3]. As a result, fiber Kerr nonlinearity effect sets an upper bound on the achievable data rate in optical fiber communications using traditional linear transmission techniques [4].

There have been extensive efforts in attempting to surpass the Kerr nonlinearity limit through several nonlinearity compensation techniques and nonlinear transmission schemes $[5,6]$. Digital-back-propagation (DBP) is an effective nonlinearity compensation method, which removes the nonlinear distortion by inverting the distorted signal at the receiver digitally, based on the fact that nonlinear impairment (signal-signal interaction, rather than signal-noise interaction is concerned) is a deterministic effect [7]. However, DBP has some serious challenges, limiting its success in practice so far.

S. T. Le, M. E. McCarthy, N. Mac Suibhne, M. A. Z. Al-Khateeb, E. Giacoumidis, N. Doran, A. D. Ellis and S. K. Turitsyn are with Aston Institute of Photonic Technologies (AIPT), School of Engineering and Applied Science, Aston Triangle, Birmingham, B4 7ET, UK (corresponding author phone: +44(0)744-702-40-09; e-mail: let1@aston.ac.uk.. This work was partially supported by the UK EPSRC programme Grants UNLOC and PEACE (EP/J017582/1 and EP/L000091/1).
Firstly, accurate DBP requires a substantial increase in digital signal processing (DSP) complexity, proportional to the number of spans. Secondly, in wavelength-division multiplexed (WDM) systems the effectiveness of DBP is significantly reduced as the neighbouring WDM channels are unknown to the compensator. In this case, only the impact of self-phase modulation, which only represents a minor part of the overall nonlinear impairment $[1,3]$, can be compensated. Finally, even though full band DBP could be achieved (with enormous complexity), it is still challenging to realize the full benefit of DBP because of polarization mode dispersion (PMD) [8] and carrier frequency uncertainty problem [9], which leads to the incorrectness in optical field reconstruction. It has been shown in [9] that even a small carrier frequency deviation of $50 \mathrm{MHz}$ can lead to a performance penalty of $\sim 2 \mathrm{~dB}$. Talking into account the fact that commercial external cavity laser (ECL) can have a frequency deviation as much as several $\mathrm{GHz}$, DBP seems to be unbeneficial for practical applications unless optical combs are employed at the transmitter [9].

Digital [10] and optical [11-13] phase conjugations (OPCs) at the mid link or installed at the transmitter [14] are other wellknown nonlinear compensation techniques that conjugate the signal phase after transmission in one segment of the link in order to achieve a net cancellation of the nonlinear phase shift using the nonlinearity generated in the second segment of the link. However, OPC modifies the transmission link by inserting a phase conjugator at the middle point of the link, and imposes significant symmetry conditions with respect to the phase conjugator, and thus, significantly reducing the flexibility in an optically routed network.

Recently, a breakthrough fibre nonlinearity compensation technique called phase-conjugated twin wave (PCTW) has been proposed by X. Liu et al $[15,16]$. PC-TW is a transponder-based technique that can be implemented with minimal additional optical hardware or DSP, providing a simple and effective solution in compensating optical fiber nonlinearity. However, PCTW halves the spectral efficiency (SE), meaning that the maximum achievable $\mathrm{SE}$ in a polarization division multiplexed (PDM) system with QPSK modulation format and PCTW scheme is only $\sim 2 \mathrm{bits} / \mathrm{s} / \mathrm{Hz}$, which is the same as those achieved in PDM BPSK transmission.

A modification of PCTW for coherent optical orthogonal frequency division multiplexing (CO-OFDM) exploring the 
Hermitian symmetry has also been proposed in [17], also at the cost of $50 \%$ overhead. To address this drawback, a flexible nonlinear compensation scheme with the insertion of phaseconjugated pilots has been proposed for CO-OFDM in [18]. This scheme allows the overhead to be adjusted (up to 50\%) according the required performance gain, which is up to $4 \mathrm{~dB}$. In addition, a dual PCTW scheme combined with quadrature pulse shaping was also proposed for single carrier systems, yielding an improvement of $\sim 1.2 \mathrm{~dB}$ [19] without any overhead.

Unfortunately, quadrature pulse shaping is required for dual PCTW, which cannot be applied effectively for multicarrier modulation formats such as CO-OFDM [20-22]. To address this issue, a phase-conjugated subcarrier coding (PCSC) scheme has been proposed in [23] by adopting the concept of dual PCTW to encoding and processing neighbouring OFDM subcarriers simultaneously. This proposed PCSC scheme can be effectively applied without any overhead and without suffering from the carrier frequency uncertainty problem. In this paper, we discuss the concept of PCSC in more details. We experimentally demonstrate the effectiveness of PCSC in WDM CO-OFDM transmissions with BPSK and QPSK modulation formats, showing that performance gains of $1.5 \mathrm{~dB}$ and $0.8 \mathrm{~dB}$ respectively (for BPSK and QPSK) can be obtained.

\section{Phase CONJUGated SUbCARRIER CODING FOR CO-OFDM TRANSMISSION}

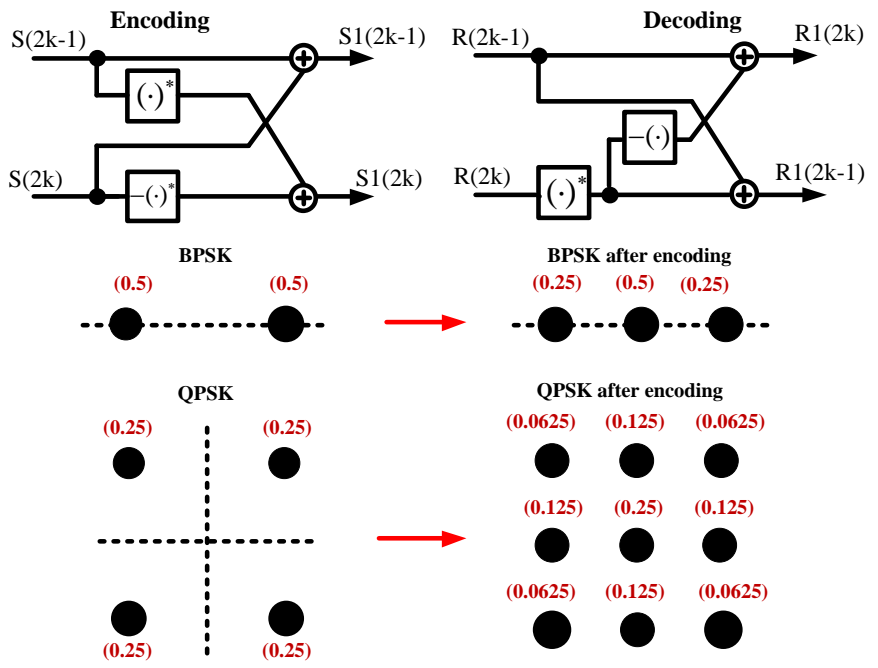

Fig. 1: Phase-conjugated subcarrier coding scheme for CO-OFDM transmission, the numbers (in red) are the probabilities of symbols in the constellation set.

In the PCSC scheme (Fig. 1) each pair of neibouring OFDM subcarriers (with the indices of $2 k-1$ and $2 k$, where $\mathrm{k}$ is an integer number) after symbol mapping are encoded before beeing fed into the IFFT block to generate the time-domain signal as:

$$
\left\{\begin{array}{l}
S_{1}(2 k-1)=S(2 k-1)+S(2 k) \\
S_{1}(2 k)=S^{*}(2 k-1)-S^{*}(2 k)
\end{array}\right.
$$

where $(.)^{*}$ stands for the complex conjugation operation.
At the receiver, before symbol demapping, the received information symbols in this subcarrier pair are decoded as:

$$
\left\{\begin{array}{l}
R_{1}(2 k-1)=R(2 k-1)+R^{*}(2 k) \\
R_{1}(2 k)=R(2 k-1)-R^{*}(2 k)
\end{array}\right.
$$

It should be noted that the PCSC can be considered as one-byone mapping scheme which does not require any overhead. The only requirement of PCSC is that the number of OFDM subcarriers is even. The PCSC scheme modifies both the constellation set and probabilities of constellation points. As shown in the Fig. 1, if the input modulation format is BPSK with equal probability $(0.5,0.5)$ for each constellation point ($1,1)$, the output constellation set will be a $3 \operatorname{ASK}(-2,0,2)$ in which the symbol "0" occurs twice as often as the two other information symbols $(-2,2)$. This indicates that $50 \%$ of BPSK OFDM subcarriers will be turned off after encoding. Similarity, if the input modulation format is QPSK, after encoding, the output constellation set will be a 9 QAM with unequal probabilities (Fig. 1), which can potentially reduce the nonlinear distortions on OFDM subcarriers due to the unequal power distribution across the OFDM band [24].

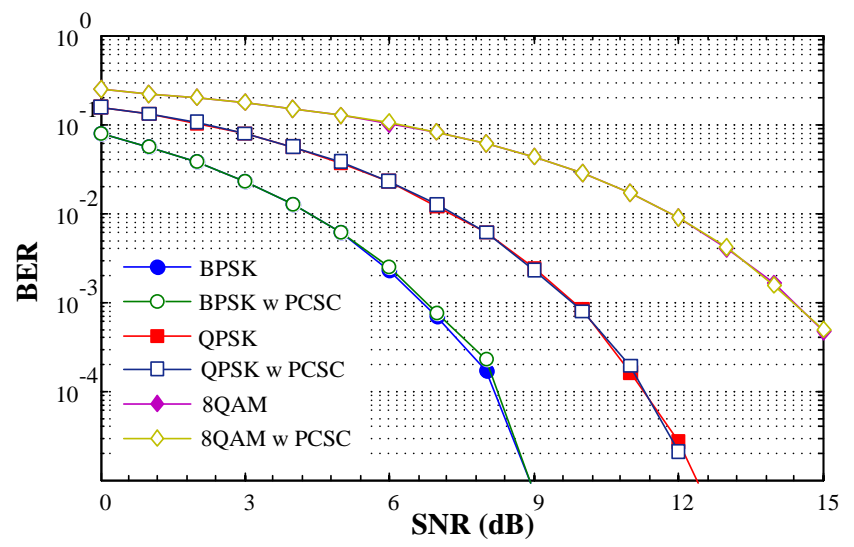

Fig. 2: Sensitivities of OFDM systems with and without PCSC in the linear channel with white Gaussian noise.

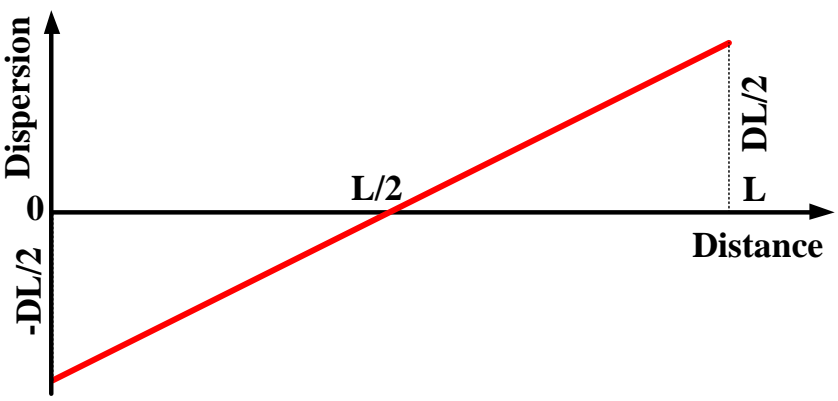

Fig. 3: Dispersion map of an optical link with $50 \%$ pre-EDC. L is the link distance and $\mathrm{D}$ is the dispersion coefficient.

The sensitivities of OFDM systems with and without PCSC scheme in the Additive White Gaussian Noise channel are compared in Fig. 2, for different modulation formats, namely BPSK, QPSK, 8QAM. It can be seen that independently of the modulation format used, PCSC gives no performance gain or penalty (the same sensitivity) in linear transmission channels. This result indicates that PCSC is ineffective for CO-OFDM 
systems if the distortions on neighbouring subcarriers are Gaussian distributed and uncorrelated. However, if the OFDM subcarrier frequency spacing is small (tens of $\mathrm{MHz}$ ) we can expect that the nonlinear phase shifts on neighbouring subcarriers will be highly correlated. Thus, potential performance gain can be achieved by encoding and processing neighbouring subcarriers simultaneously at the transmitter and receiver. In order to enhance the similarity of nonlinear distortions on neighbouring OFDM subcarriers, pre-EDC is applied in this work to create a dispersion-symmetry along the transmission link as shown in Fig. 3 [16].

\section{SimULATION}

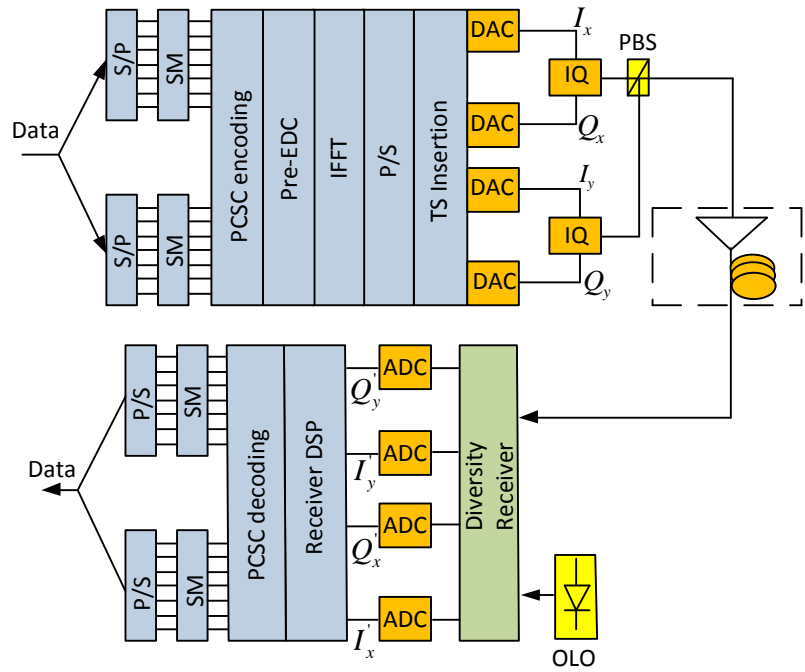

Fig. 4. Block diagram of PDM CO-OFDM transmissions with PCSC. S/P: serial/parallel conversion, P/S: parallel/serial conversion, SM: symbol mappings, TS: training symbol, DAC: digital-to-analog converter, ADC: analog-to-digital converter, I/Q: I/Q modulator, PBS: polarization beam spliter, OLO: optical local oscilator.

As a proof of concept, we first conducted a simulation of the PCSC scheme in a single channel 80Gbaud PDM CO-OFDM transmission system with BPSK and QPSK modulation formats. It should be noted that the choice of signal bandwidth is not critical here. The simulation setup is shown in the Fig. 4. The data stream was first divided into $\mathrm{x}$ - and y-polarizations, each of which was then mapped onto 1000 subcarriers using BPSK and QPSK modulation formats and subsequently transferred to the time domain by an IFFT of size 2048 while zeros occupying the remainder. The OFDM useful symbol duration was $12 \mathrm{~ns}$ and a cyclic prefix of $0.4 \mathrm{~ns}$ was added for polarization mode dispersion (PMD) compensation. The net bit-rate (after extracting $7 \%$ FEC) is $150 \mathrm{~Gb} / \mathrm{s}$ and $300 \mathrm{~Gb} / \mathrm{s}$ when BPSK and QPSK are adopted. The long-haul fiber link comprised $80-\mathrm{km}$ spans of standard single mode fibre (SSMF) with a loss parameter of $0.2 \mathrm{~dB} / \mathrm{km}$, nonlinearity coefficient of $1.22 / \mathrm{W} / \mathrm{km}$, dispersion of $16 \mathrm{ps} / \mathrm{nm} / \mathrm{km}$ and PMD coefficient of $0.1 \mathrm{ps} / \mathrm{km}^{1 / 2}$. The span loss was compensated by Erbiumdoped fibre amplifiers with $16 \mathrm{~dB}$ of gain and $6 \mathrm{~dB}$ noise figure. The amplified spontaneous emission noise is added inline to ensure that the interaction between signal and noise is correctly captured [25]. The transmitter and receiver lasers had the same linewidth of $100 \mathrm{kHz}$. The simulated time window contained 500 OFDM symbols $\left(10^{6}\right.$ bits for QPSK). The DSP at the receiver includes chromatic dispersion compensation using a frequency domain equalizer with overlap-and-save method, channel estimation and equalization with the assistance of initial training sequence (2 training symbols every 100 symbols) using zero forcing estimation with MIMO processing, common phase error compensation with the insertion of quasi-pilot subcarriers [26, 27] (4 pilots every OFDM symbol) and symbol detection. The system performance is evaluated using the Q-factor derived directly from the BER [28].

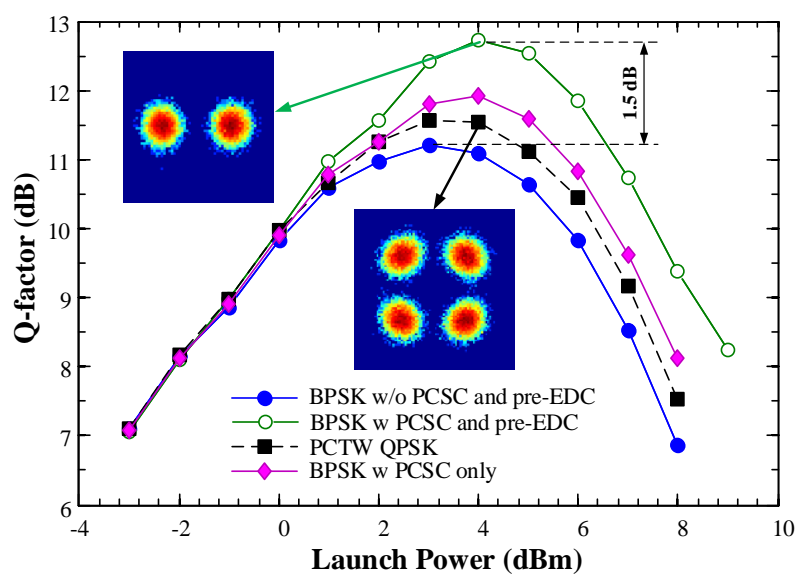

Fig. 5. Q-factor as a function of the launch power in $150 \mathrm{~Gb} / \mathrm{s}$ PDM COOFDM system with and without PCSC, the transmission distance is $8000 \mathrm{~km}$
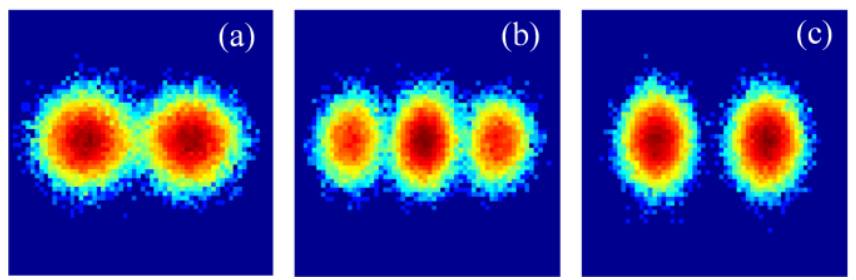

Fig. 6. Constellation diagrams on $\mathrm{x}$-polarization, $8 \mathrm{dBm}$ of the launch power, a) - without PCSC, b, c) with PCSC, before and after decoding.

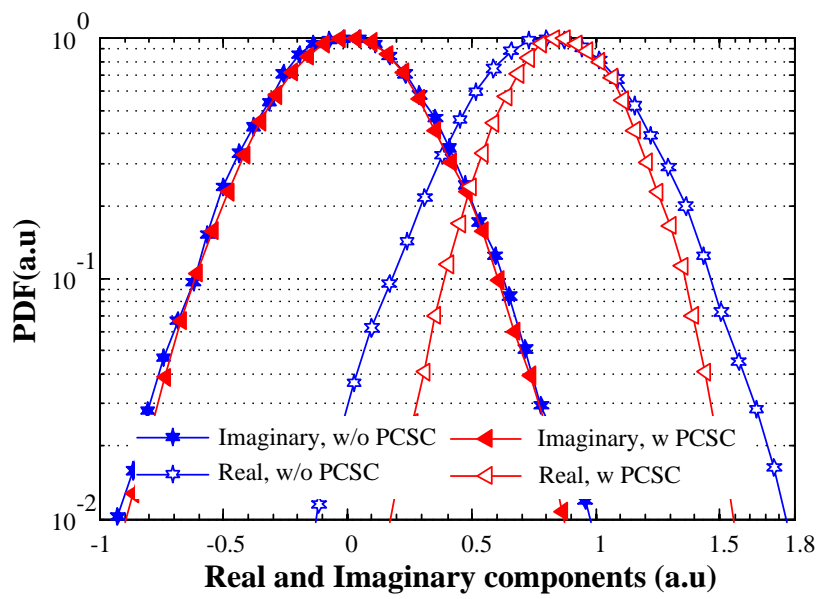

Fig. 7. PDF of real and imaginary components for the "1" symbol in systems with and without the PCSC, the launch power was $7 \mathrm{dBm}$.

Performances of the $150 \mathrm{~Gb} / \mathrm{s}$ PDM CO-OFDM systems with and without the PCSC scheme (with and without pre-EDC) are compared in the Fig. 5. In this figure, the performance of PCTW technique with QPSK modulation format providing the 
same SE ( 2 bits/s/Hz) is also presented. As PCTW halves the $\mathrm{SE}$, despite the effective nonlinear noise cancellation effect, PCTW with QPSK modulation format gives only around $0.5 \mathrm{~dB}$ advantage over the traditional BPSK PDM CO-OFDM transmission scheme. On the other hand, when the PCSC coding scheme combined with pre-EDC is applied, a performance improvement of $1.5 \mathrm{~dB}$ can be achieved without reducing the SE. When PCSC is applied without $50 \%$ preEDC, a performance gain of $\sim 0.7 \mathrm{~dB}$ is observed. This result clearly indicates the benefit of pre-EDC in the proposed transmission scheme, which enhances the total gain to $1.5 \mathrm{~dB}$. Interestingly, a nonlinear noise squeezing effect was observed (Fig. 6) in a similar manner as in single carrier system with real-valued signal and the symmetrical dispersion map [29]. Without PCSC, the real and imaginary parts of each constellation point have the same distribution. However, with PCSC and the optimized pre-EDC, the PDF of the real part of each constellation point is significantly narrowed. This nonlinear noise squeezing effect significantly reduces the BER in a transmission system using BPSK modulation format.

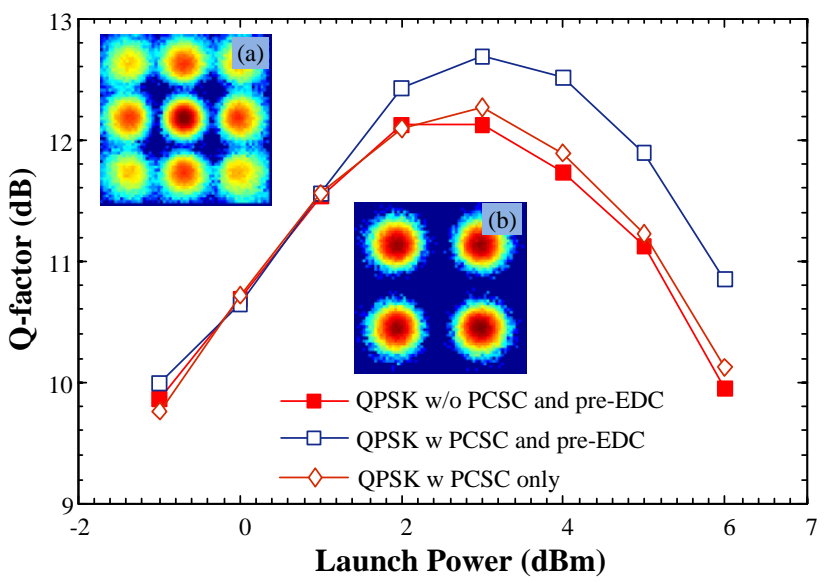

Fig. 8. Q-factor as a function of the launch power in $300 \mathrm{~Gb} / \mathrm{s}$ PDM COOFDM system with and without PCSC and constellation diagrams (before (a) and after (b) decoding) at $4 \mathrm{dBm}$, after $3200 \mathrm{~km}$ of transmission distance.

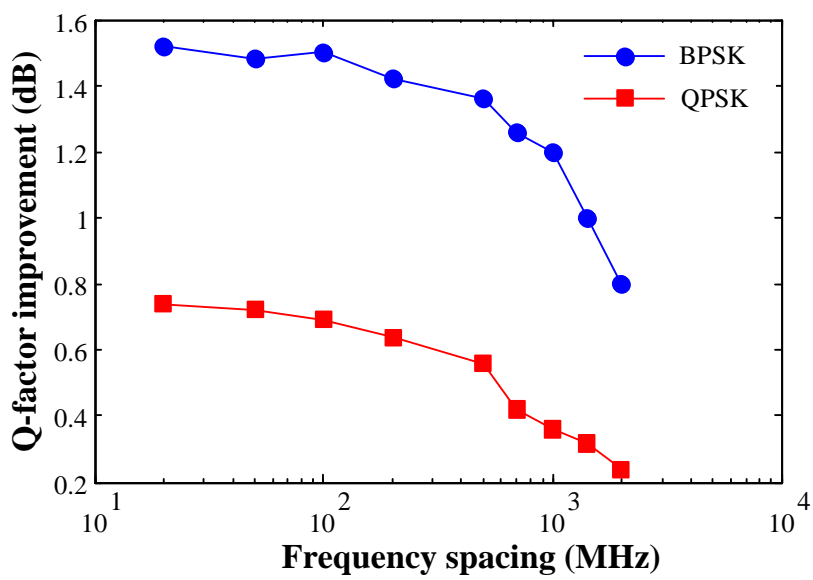

Fig. 9. Performance gain in systems with PCSC as a function of the frequency spacing for different modulation formats.

When the PCSC scheme combined with the optimized preEDC is applied for $300 \mathrm{~Gb} / \mathrm{s}$ QPSK PDM CO-OFDM system, a performance improvement of around $0.7 \mathrm{~dB}$ is achieved, as shown in the Fig. 8. This result clearly indicates that the proposed PCSC scheme also effectively mitigates the nonlinear distortions on OFDM subcarriers when QPSK modulation format is adopted. However, as QPSK cannot take the advantage of the nonlinear noise squeezing effect, the performance improvement in this case is only a half of those achieved with BPSK modulation format. In addition, without $50 \%$ pre-EDC, PCSC does not provide a significant improvement in the system's performance. This result confirms the benefit of pre-EDC in applying the proposed coding scheme (both for BPSK and QPSK modulation formats).

The performance gain offered by PCSC and pre-EDC as a function of frequency spacing is shown in Fig. 9 for BPSK and QPSK. Herein, the signal bandwidth is kept at $80 \mathrm{GHz}$, the number of OFDM subcarrier and the IFFT size are reduced accordingly to increase the OFDM subcarriers frequency spacing. For example, the number of OFDM subcarrier and the IFFT size were set to 160 and 512 to increase the subcarriers spacing to $500 \mathrm{MHz}$. As expected, the performance gain decreases with the increasing of the frequency spacing. If the frequency spacing is comparable with the FWM bandwidth $(\sim 1 \mathrm{GHz})$, the performance gain in QPSK system becomes negligible $(\sim 0.2 \mathrm{~dB})$. This result clearly indicates that the OFDM frequency spacing should be kept small in order to take the advantage of PCSC scheme.

\section{EXPERIMENTAL DEMONSTRATION}

The experimental set-up is shown in Fig. 10. It comprised three standard DFBs on $25 \mathrm{GHz}$ grid which were substituted in turn by a $100 \mathrm{kHz}$ linewidth laser. Additional loading channels (10 GHz of bandwidth) were generated using an ASE source which were spectrally shaped using a wavelength selective switch (WSS) [30]. The twenty loading channels were spread symmetrically around the test wavelengths so that the total bandwidth of the transmitted signal was $0.575 \mathrm{THz}$. The transmission path was re-circulating loop consisting of a single span $100 \mathrm{~km}$ Sterlite OH-LITE (E) fiber, having around $19 \mathrm{~dB}$ insertion loss. A gain flattening filter (GFF) was placed in the mid stage of the EDFA. After propagation the center channel was coherently detected. The received electrical signals were then sampled by a real-time oscilloscope at $80 \mathrm{GS} / \mathrm{s}$ and processed offline in MATLAB.

The OFDM signals (400 symbols each of $20.48 \mathrm{~ns}$ length, $2 \%$ cyclic prefix) encoded with BPSK and QPSK modulation formats were generated offline in MATLAB using an IFFT size of 512, where 210 subcarriers were filled with data and the remainder zeros giving a line rate of $10 \mathrm{~Gb} / \mathrm{s}$ and $20 \mathrm{~Gb} / \mathrm{s}$ $(9.1 \mathrm{~Gb} / \mathrm{s}$ and $18.2 \mathrm{~Gb} / \mathrm{s}$ after cyclic prefix and FEC overhead are removed) for BPSK and QPSK modulation formats respectively. The DSP at the receiver included synchronization, $x$ - and $y$-polarizations combination using the maxima-ratio combining method [31], frequency offset compensation, chromatic dispersion compensation using an overlapped frequency domain equalizer with overlap-and-save method, channel estimation and equalization with the assistance of initial training sequence (2 training symbols every 100 symbols), phase noise compensation with the help 8 

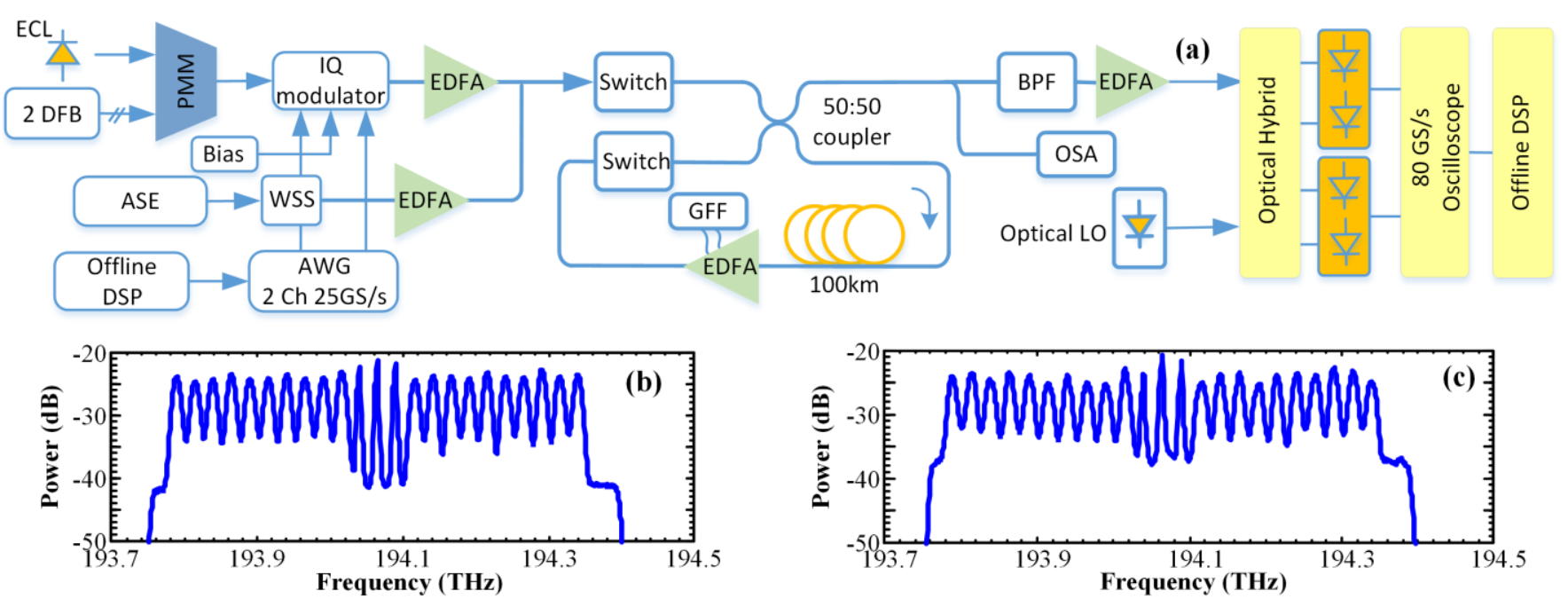

Fig. 10 (a) - Schematic of experimental setup of WDM CO-OFDM transmission with PCPs for fibre nonlinearity compensation. ECL: external cavity laser, PMM: polarization maintaining multiplexer, WSS: Wavelength Selective Switch, DFB: distributed feedback laser, BPF: band-pass filter (optical), GFF: gain flatten filter, OSA: optical spectrum analyser, LO: local oscillator. (b) - optical spectrum after the transmitter. (c) - optical spectrum after $2400 \mathrm{~km}$ of transmission distance.

pilot subcarriers, and symbol detection. The system performance was evaluated directly from the BER by processing 10 recorded traces $\left(\sim 10^{6}\right.$ bits $)$, the results also are expressed as a Q-factor [32].

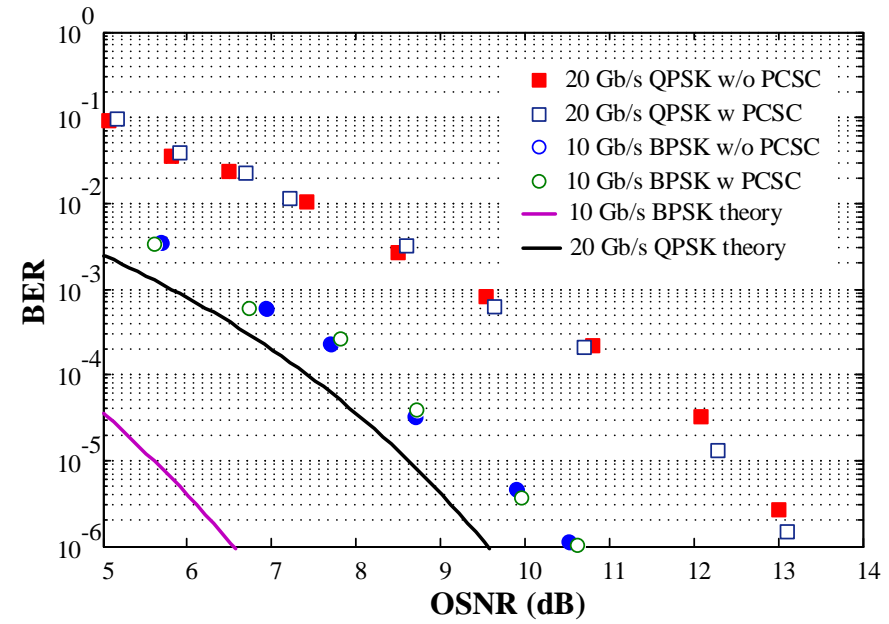

Fig. 11. Back-to-back performance of OFDM systems with and without PCSC with BPSK and QPSK modulation formats.
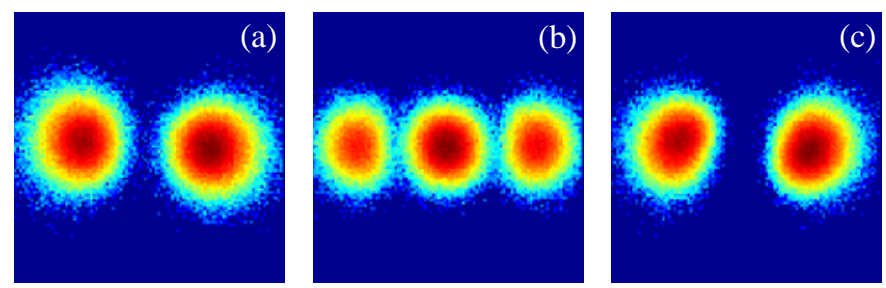

Fig. 12. Received constellation diagrams of the center channel at $4000 \mathrm{~km}$ of transmission distance, the launch power/channel was $-3 \mathrm{dBm}$, a) - without PCSC, b, c) with PCSC, before and after decoding.

The BER as a function of optical signal-to-noise ratio (OSNR) are compared in the back-to-back case for systems with and without PCSC in the Fig. 11. In a good agreement with the simulation results presented in Fig. 2, the BER remains the same in systems with and without PCSC. This result clearly confirms that PCSC does not affect the system sensitivity in the back-to-back case. It should also be noted in Fig. 11 that the implementation penalty (at the BER level of $10^{-3}$ ) is around $4 \mathrm{~dB}$.

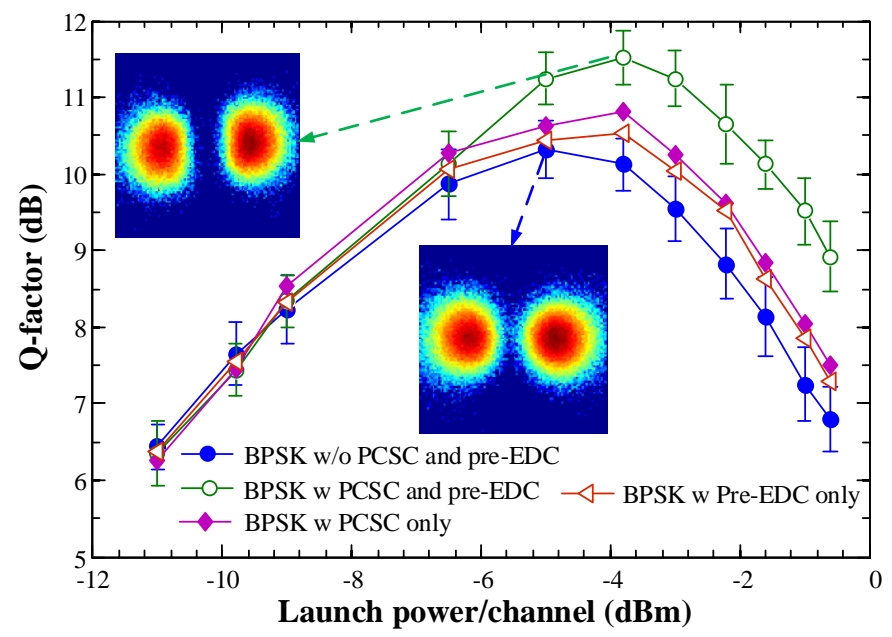

Fig. 13. Q-factor as a function of the launch power/channel for the center channel in BPSK WDM CO-OFDM systems with and without PCSC, the transmission distance is $6000 \mathrm{~km}$.

The received constellation diagrams in BPSK transmission with and without PCSC (with pre-EDC) after $4000 \mathrm{~km}$ are shown in Fig. 12 for a launch power/channel of $-3 \mathrm{dBm}$. It is clearly that the received signal quality is significantly increased when PCSC is with 50\% pre-EDC is applied. This result confirms that the fiber nonlinearity impairment is effectively mitigated by encoding and processing neighbouring subcarriers by the PCSC scheme. Herein, the nonlinear noise squeezing effect can also be observed as the nonlinear distortion in the imaginary component of the received information symbol tends to be bigger than those of the real component.

The Q-factor as a function of the launch power in BPSK transmissions with and without PCSC and pre-EDC is plotted 
in Fig. 13 for a transmission distance of $6000 \mathrm{~km}$. The constellation diagrams at the optimum launch power for both cases are also included. In Fig. 13, a performance improvement of around $1.5 \mathrm{~dB}$ is observed, which is equivalent with the simulation result plotted in Fig. 5 for single channel transmission. This result indicates that PCSC with preEDC is also effective in compensating the nonlinear distortions due to cross phase modulation as long as the OFDM frequency spacing is small. This result confirms that PCSC is effective in both single and WDM transmission configurations.

It should be noted that without pre-EDC, PCSC only provides $\sim 0.7 \mathrm{~dB}$ performance gain. Moreover, pre-EDC without PCSC shows a slightly worse performance improvement $(\sim 0.5 \mathrm{~dB})$. As a result, a combination of PCSC and pre-EDC is necessary to achieve the full benefit of the proposed nonlinear mitigation scheme.

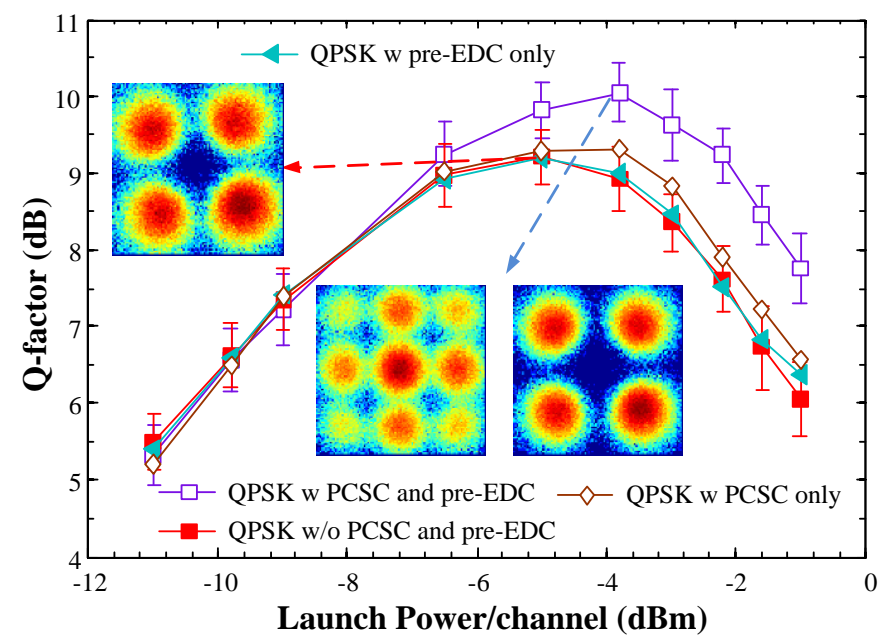

Fig. 14. Q-factor as a function of the launch power/channel for the center channel in QPSK WDM CO-OFDM systems with and without PCSC, the transmission distance is $4000 \mathrm{~km}$.

The similar result for QPSK WDM CO-OFDM transmission is shown in Fig. 14, at a transmission distance of $4000 \mathrm{~km}$. The performance enhancement observed is around $0.8 \mathrm{~dB}$ when PCSC combined with pre-EDC is applied, confirming that PCSC can also be effectively applied for a high SE modulation format such as QPSK. In Fig. 14, pre-EDC without PCSC does not improve the system performance. Similarly, without $50 \%$ pre-EDC, PCSC does not provide a significant performance gain, which agrees well with the simulation result presented in Fig. 8. As a consequence, PCSC should be combined with preEDC to achieve the best performance for both BPSK and QPSK transmissions. As we explained before, the nonlinear noise squeezing effect is not beneficial to a quadrature modulation format such as QPSK. As a result, the performance gain in QPSK transmission is smaller than those obtained in BPSK transmission.

\section{CONCLUSION}

We have showed that the fiber nonlinearity impairments in CO-OFDM transmission can be mitigated by processing neighboring subcarriers simultaneously using the PCSC scheme. This coding scheme is very simple and can be effectively combined with pre-EDC to achieve a performance improvement up to $1.5 \mathrm{~dB}$. In addition, it can be effectively applied in both single polarization and PDM systems, in both single channel and WDM systems without suffering from carrier uncertainty problem.

\section{ACKNOWLEDGMENT}

This work was partially supported by the UK EPSRC programme Grants UNLOC and PEACE (EP/J017582/1 and $\mathrm{EP} / \mathrm{L} 000091 / 1)$ and the European Commission's 7th Framework Programme FP/2007-2013 grants 258033 (MODE-GAP), 318137 (DISCUS) and 318415 (FOX-C), and the support of Sterlite Technologies and Finisar is gratefully acknowledged.

\section{REFERENCES}

[1] R. Essiambre, G. Kramer, P. J. Winzer, G. J. Foschini, and B. Goebel, "Capacity Limits of Optical Fiber Networks," Journal of Lightwave Technology, vol. 28, pp. 662-701, 2010.

[2] P. P. Mitra, Stark, Jason B., "Nonlinear limits to the information capacity of optical fibre communications," Nature, vol. 411, pp. 1027-1030, 2001.

[3] A. D. Ellis, Z. Jian, and D. Cotter, "Approaching the Non-Linear Shannon Limit," Journal of Lightwave Technology, vol. 28, pp. 423433, 2010.

[4] S. T. Le, J. E. Prilepsky, and S. K. Turitsyn, "Nonlinear inverse synthesis for high spectral efficiency transmission in optical fibers," Opt. Express, pp. 26720-26741, 2014.

[5] D. Rafique, J. Zhao, and A. D. Ellis, "Digital back-propagation for spectrally efficient WDM 112 Gbit/s PM m-ary QAM transmission," Optics Express, vol. 19, pp. 5219-5224, 2011/03/14 2011.

[6] J. E. Prilepsky, S. A. Derevyanko, and S. K. Turitsyn, "Nonlinear spectral management: Linearization of the lossless fiber channel," Optics Express, vol. 21, pp. 24344-24367, 2013/10/07 2013.

[7] E. Ip and J. M. Kahn, "Compensation of Dispersion and Nonlinear Impairments Using Digital Backpropagation," Journal of Lightwave Technology, vol. 26, pp. 3416-3425, 2008/10/15 2008.

[8] G. Liga, T. Xu, A. Alvarado, R. I. Killey, and P. Bayvel, "On the performance of multichannel digital backpropagation in highcapacity long-haul optical transmission," Optics Express, vol. 22, pp. 30053-30062, 2014/12/01 2014.

[9] N. Alic, E. Myslivets, E. Temprana, B. P. P. Kuo, and S. Radic, "Nonlinearity Cancellation in Fiber Optic Links Based on Frequency Referenced Carriers," Journal of Lightwave Technology, vol. 32, pp. 2690-2698, 2014.

[10] X. Chen, X. Liu, S. Chandrasekhar, B. Zhu, and R. W. Tkach, "Experimental demonstration of fiber nonlinearity mitigation using digital phase conjugation," in Optical Fiber Communication Conference and Exposition (OFC/NFOEC), 2012 and the National Fiber Optic Engineers Conference, 2012, pp. 1-3.

[11] S. L. Jansen, D. Van den Borne, B. Spinnler, S. Calabro, H. Suche, P. M. Krummrich, et al., "Optical phase conjugation for ultra long-haul phase-shift-keyed transmission," Journal of Lightwave Technology, vol. 24, pp. 54-64, 2006.

[12] D. M. Pepper and A. Yariv, "Compensation for phase distortions in nonlinear media by phase conjugation," Optics Letters, vol. 5, pp. 5960, 1980/02/01 1980.

[13] I. Phillips, M. Tan, M. F. Stephens, M. McCarthy, E. Giacoumidis, S. Sygletos, et al., "Exceeding the Nonlinear-Shannon Limit using Raman Laser Based Amplification and Optical Phase Conjugation," in Optical Fiber Communication Conference, San Francisco, California, 2014, p. M3C.1.

[14] S. Watanabe, S. Kaneko, and T. Chikama, "Long-Haul Fiber Transmission Using Optical Phase Conjugation," Optical Fiber Technology, vol. 2, pp. 169-178, 4// 1996. 
[15] X. Liu, R. A. Chraplyvy, P. J. Winzer, W. R. Tkach, and S. Chandrasekhar, "Phase-conjugated twin waves for communication beyond the Kerr nonlinearity limit," Nat Photon, vol. 7, pp. 560-568, 2013.

[16] X. Liu, S. Chandrasekhar, P. J. Winzer, R. W. Tkach, and A. R. Chraplyvy, "Fiber-Nonlinearity-Tolerant Superchannel Transmission via Nonlinear Noise Squeezing and Generalized Phase-Conjugated Twin Waves," Journal of Lightwave Technology, vol. 32, pp. 766$775,2014$.

[17] X. Yi, X. Chen, D. Sharma, C. Li, M. Luo, Q. Yang, et al., "Digital coherent superposition of optical OFDM subcarrier pairs with Hermitian symmetry for phase noise mitigation," Optics Express, vol. 22, pp. 13454-13459, 2014/06/02 2014.

[18] S. T. Le, M. E. McCarthy, N. M. Suibhne, A. D. Ellis, and S. K. Turitsyn, "Phase-conjugated Pilots for Fibre Nonlinearity Compensation in CO-OFDM Transmission," presented at the ECOC, Cannes, France, paper We.2.3.1, 2014.

[19] T. Yoshida, T. Sugihara, K. Ishida, and T. Mizuochi, "Spectrallyefficient Dual Phase-Conjugate Twin Waves with Orthogonally Multiplexed Quadrature Pulse-shaped Signals," in Optical Fiber Communication Conference, San Francisco, California, 2014, p. M3C.6.

[20] W. Shieh, X. Yi, Y. Ma, and Q. Yang, "Coherent optical OFDM: has its time come? [Invited]," Journal of Optical Networking, vol. 7, pp. 234-255, 2008/03/01 2008.

[21] W. Shieh and C. Athaudage, "Coherent optical orthogonal frequency division multiplexing," Electronics Letters, vol. 42, pp. 587-589, 2006.

[22] J. Zhao and A. D. Ellis, "A Novel Optical Fast OFDM with Reduced Channel Spacing Equal to Half of the Symbol Rate per Carrier," in Optical Fiber Communication Conference, San Diego, California, 2010, p. OMR1.

[23] S. T. Le, E. Giacoumidis, N. Doran, A. D. Ellis, and S. K. Turitsyn, "Phase-conjugated Subcarrier Coding for Fibre Nonlinearity Mitigation in CO-OFDM Transmission," presented at the ECOC, Cannes, France, paper We.3.3.2, 2014.

[24] S. T. Le, K. Blow, and S. Turitsyn, "Power pre-emphasis for suppression of FWM in coherent optical OFDM transmission," Optics Express, vol. 22, pp. 7238-7248, 2014/03/24 2014.

[25] D. Rafique and A. D. Ellis, "Impact of signal-ASE four-wave mixing on the effectiveness of digital back-propagation in $112 \mathrm{~Gb} / \mathrm{s} \mathrm{PM}-$ QPSK systems," Optics Express, vol. 19, pp. 3449-3454, 2011/02/14 2011.

[26] S. T. Le, T. Kanesan, M. McCarthy, E. Giacoumidis, I. Phillips, M. F. Stephens, et al., "Experimental Demonstration of Data-dependent Pilot-aided Phase Noise Estimation for CO-OFDM," in Optical Fiber Communication Conference, San Francisco, California, 2014, p. Tu3G.4.

[27] L. Son Thai, T. Kanesan, E. Giacoumidis, N. J. Doran, and A. D. Ellis, "Quasi-Pilot Aided Phase Noise Estimation for Coherent Optical OFDM Systems," Photonics Technology Letters, IEEE, vol. 26, pp. 504-507, 2014.

[28] S. T. Le, K. J. Blow, V. K. Menzentsev, and S. K. Turitsyn, "Comparison of numerical bit error rate estimation methods in 112Gbs QPSK CO-OFDM transmission," in Optical Communication (ECOC 2013), 39th European Conference and Exhibition on, 2013, pp. 1-3.

[29] X. Liu, S. Chandrasekhar, P. J. Winzer, R. W. Tkach, and A. R. Chraplyvy, "406.6-Gb/s PDM-BPSK superchannel transmission over 12,800-km TWRS fiber via nonlinear noise squeezing," in Optical Fiber Communication Conference and Exposition and the National Fiber Optic Engineers Conference (OFC/NFOEC), 2013, 2013, pp. $1-3$.

[30] M. E. McCarthy, N. M. Suibhne, S. T. Le, P. Harper, and A. D. Ellis, "High Spectral Efficiency Transmission Emulation for Non-Linear Transmission Performance Estimation for High Order Modulation Formats," presented at the ECOC, Cannes, France, paper P.5.7, 2014.

[31] K. Kikuchi and S. Tsukamoto, "Evaluation of Sensitivity of the Digital Coherent Receiver," Journal of Lightwave Technology, vol. 26, pp. 1817-1822, 2008.
[32] S. T. Le, K. Blow, V. Mezentsev, and S. Turitsyn, "Bit Error Rate Estimation Methods for QPSK CO-OFDM Transmission," Journal of Lightwave Technology, vol. PP, pp. 1-1, 2014. 